\title{
Evaluación de la gestión institucional y la calidad del servicio educativo en las instituciones educativas del distrito de Huaral - Lima, 2018
}

\section{Evaluation of the institutional management and quality of the educational service in the educational institutions of the district of Huaral - Lima, 2018}

\author{
Rosendo Teodoro Rivas Tupia ${ }^{1}$, Abelardo Rodolfo Campana Concha ${ }^{2}$
}

\begin{abstract}
RESUMEN
Objetivo: Analizar las variables Gestión Institucional y Calidad del Servicio Educativo, en el contexto educativo de las Instituciones Educativas del distrito de Huaral - Lima, 2018. Material y Métodos: Se planteó el análisis correlacional para lo cual se elaboró una encuesta para cada variable, cuyos resultados fueron determinados por el programa estadistico SPSS, con el uso de la fórmula de Rho de Spearman con un margen de error al 5\% para presentar las conclusiones. Resultados: La hipótesis general estableció una correlación de Rho $=0,822$ lo cual pone en manifiesto que la gestión institucional considerado a veces adecuado esta relacionada a la calidad del servicio educativo. Las seis hipótesis específicas identificaron correlaciones de $\mathrm{Rho}=0,561$, Rho $=0,640, \mathrm{Rho}=0,562$, Rho $=0,472$, Rho $=0,757, \mathrm{Rho}=0,621$ con los cuales se pudo comprender que la motivación para el aprendizaje, la convivencia escolar, la cooperación con entidades productivas, la política de inclusión, clima institucional y participación identificados a perspectiva de los estudiantes como a veces adecuado, repercuten significativamente con la infraestructura, actuación del personal y la organización, las cuales pertenecen a las dimensiones de la calidad del servicio educativo. Conclusión: La gestión institucional no cumple con las espectatívas de los estudiantes, lo cual disminuye las posibilidades de progreso pero en la calidad del servicio.
\end{abstract}

Palabras clave: Gestión institucional, calidad, servicio educativo, motivación, convivencia escolar, cooperación, inclusión, clima institucional, participación.

\begin{abstract}
Objective: To analyze the variables Institutional Management and Quality of the Educational Service, in the educational context of the Educational Institutions of the district of Huaral - Lima, 2018. Material and Methods: The correlational analysis was proposed for which a survey was prepared for each variable, whose results were determined by the SPSS statistical program, with the use of Spearman's Rho formula with a margin of error of $5 \%$ to present the conclusions. Results: The general hypothesis established a correlation of Rho $=0,822$ which shows that the institutional management considered sometimes appropriate is related to the quality of the educational service. The six specific hypotheses identified correlations of $R h o=0,561$, $R h o=0,640$, $R h o=0,562$, $R h o=0,472$, Rho $=0,757$, Rho $=0,621$ with which it was possible to understand that the motivation for learning, the school coexistence, cooperation with productive entities, the inclusion policy, institutional climate and participation identified in the students' perspective as sometimes adequate, have a significant impact on the infrastructure, staff performance and organization, which belong to the dimensions of the quality of the educational service. Conclusion: Institutional management does not meet the expectations of students, which reduces the chances of progress but in the quality of service
\end{abstract}

Keywords: Institutional management, quality, educational service, motivation, school coexistence, cooperation, inclusion, institutional climate, participation.

\section{INTRODUCCIÓN}

Durante los últimos años, se observa un desarrollo universalizado de evaluación que va más allá del ámbito de los aprendizajes. Se ha ido dando un traslado de la evaluación educativa desde un carácter que es exclusivo de cada docente en su aula hacia otro espacio de carácter más público y general, consiguiendo de esta manera hacer más efectiva la labor educativa y permitiendo apreciar mejor el desarrollo de los aprendizajes en todos los niveles.

Analizar la gestión en cada institución educativa depende de IPEBA, que según SINEACE (2014) es la entidad pública y autónoma perteneciente a la suya, cuyo objetivo es analizar la institución en base a siete aspectos: equidad, interculturalidad, diversidad, relevancia, pertinencia, eficacia y eficiencia las cuales según las calificaciones determinará la calidad necesaria para admitirle la acreditación correspondiente. Sin embargo, hasta el 2017, solo 18 instituciones educativas demostraron, en sus evaluaciones, que son de calidad y otros 557 en todo el país están trabajando para cumplir con los estándares nacionales, incluso es importante enfatizar que la mayoría de estas instituciones son privadas, poniendo en evidencia la realidad de las estatales.

Escale (2017) con sus más recientes estadísticas nos brinda un panorama más amplio de la realidad educativa. En cuanto a los aprendizajes alcanzados, el nivel primario en cuanto a la comprensión de textos el $50.9 \%$ de la población urbana consigue ello, mientras que solo un $16.5 \%$ de los estudiantes en zonas rurales. Este análisis también se extendió en la matemática en donde solo el $36.6 \%$ de la zona urbana aprende idóneamente, mientras que la situación en la zona rural es representada por el $17.3 \%$. En cuanto al nivel secundaria, las estadísticas demuestran que el $14.3 \%$ comprende textos idóneamente, el $11.5 \%$ con matemáticas y $15.0 \%$ Historia, Geografía y Economía.

Por lo dicho anteriormente, es importante destacar lo señalado en un informe realizado por el diario Gestión, indica que el Banco Interamericano de Desarrollo (BID), el Perú, hacia 2015 , con US\$20,114 por estudiante, se estableció como uno de los países con menos inversión de US\$50,000 en sus estudiantes de 6 a 15 años, sabiendo que aumentar el presupuesto en educación traería como consecuencia elevar un mejor nivel de educación.

Sumado a ello, Guadalupe et. al (2017) agrega que según Cooperación y el Desarrollo Económicos (OCDE) estamos muy por debajo del promedio de inversión a comparación de los países de la región y miembros de esta organización encargada de velar por la cantidad financiera que se necesita para la educación.

\footnotetext{
1 Estudiante de Maestría de la Universidad Nacional Mayor de San Marcos.

2 Docente de Educación de la Universidad Nacional Mayor de San Marcos. Recibido: 13/03/2019 Aprobado: 31/03/2019
} 
Si adentramos a los aspectos de infraestructura, podemos darnos una idea de la realidad en la cual se ofrece el servicio educativo, según los informes actualizados de ESCALE, en las zonas urbanas hasta el 2016 solo $23,6 \%$ tenían locales en buen estado, cifras que incrementarían para bien en 2017 ya que un $27,2 \%$ contaba con las infraestructuras idóneas para estudiar. En la zona rural, la situación es diferente puesto que hasta hace dos años el 19,3\% instituciones contaban con un buen local, también se presentó una leve mejora ya que el 2016 solo el $17,6 \%$ contaba con ese privilegio. Aunque las cifras muestran crecimiento, no pasa del $50 \%$ lo que nos demuestra que alcanzar la calidad se está convirtiendo en una tarea complicada, puesto estas escuelas, en especial en las zonas rurales no cuentan ni siquiera con los servicios básicos ya que hasta el 2017 , solo el $21,9 \%$ contaba con agua luz y desagüe.

Los docentes también son parte importante para la gestión institucional y la calidad del servicio educativo, según el Consejo Nacional de Educación (2016) indica que los docente de inicial, primaria y secundaria, entre los años 2009 y 2014, admitieron que como mínimo una vez al año forman parte de un curso de actualización, los cuales en su mayoría eran ofrecidos por alguna institución del Estado, asimismo las dos terceras parte de ellos consideran prudente capacitarse más en estrategias y didácticas de las áreas de aprendizaje.

La evaluación de instituciones educativas simboliza un camino para llegar a un objetivo: lograr instituciones educativas con mayor eficacia y de calidad basada en una organización que permita evaluar y tomar decisiones en un proceso sistemático y constante que permita una evolución progresiva en la consecución de las metas y de esta manera generar un progreso en la educación expresado en la calidad institucional. Entretanto, los problemas que enfrenta la educación peruana afectan a todo el ámbito educativo, que involucra elementos como el currículo, la evaluación, la cobertura, el bienestar estudiantil, el profesorado y a la calidad educativa (que es un término muy amplio y que a su vez abarca otros aspectos).

Areche (2013), señala que la acción inteligente para gestionar un centro educativo incide favorablemente en la calidad educativa. Sin embargo, considera que una institución debe plantearse metodologías más humanas, que permitan el desarrollo de todos sus integrantes, algo que es muy cierto, el trato humano es fundamental para la comprensión, el intercambio de ideas, pensando siempre en la satisfacción del estudiante.

Por su parte, Ropa (2014) encuentra que la calidad en los servicios es necesario para la productividad de la mayoría de las instituciones, entre ellas las educativas, puesto que tiene que racionar los recursos de forma idónea y aprovechar la capacidad de sus integrantes (potencial humano), de esta manera el servicio podrá abarcar las necesidades del cliente final que en la realidad educativa hace referencia a los estudiantes. Por otro lado, agrega que el mejoramiento de la calidad educativa debe incluir los aportes de las personas, haciendo uso de los recursos, siguiendo los procedimientos necesarios en base a la eficacia para alcanzar los objetivos formulados por la institución, cuyo planteamiento refuerza que todos los partícipes de la institución fomentan, ayudan y participan en la calidad de la educación.

Este estudio se justifica en el aspecto teórico, puesto que existen estudios e investigaciones limitadas que abordan de forma conjunta la evaluación institucional y su relación con la calidad educativa en las instituciones educativas. Ante la necesidad de ampliar y profundizar, este estudio buscará reconocer los beneficios de la evaluación institucional como componente que aporta al mejoramiento de la calidad educativa de un centro educativo, teniendo en cuenta los problemas de infraestructura inadecuada, incumplimiento de las metas educativas, falta de capacitaciones docentes y ausencia de liderazgo de los directivos que se presentan en las escuelas.

En cuanto a la justificación práctica, damos cuenta de la necesidad de contribuir con la evaluación educativa, y las ciencias relacionadas a la educación, cuyos aportes incluidos en la investigación permitan fortalecer estas disciplinas para crear herramientas que potencien la toma de decisiones en el ámbito de la evaluación de la calidad educativa. A su vez, ofrecerá estrategias y herramientas actuales que contribuyen a mejorar la evaluación institucional y a mejorar las estrategias para lograr un progreso adecuado.

Por otra lado, este estudio también está justificado metodológicamente, puesto que pretende establecer un conjunto de estrategias, métodos y procedimientos que sirvan de modelo para que las instituciones educativas puedan potenciar el nivel de liderazgo, recursos materiales, personal, planificación estratégica y procesos, ya que el éxito de la institución educativa surge de una adecuada evaluación institucional, que utilice métodos e instrumentos actualizados, que se puedan aplicar a la Institución educativa para lograr una calidad educativa óptima. Por tanto el objetivo central de la investigación consistió en analizar la relación que existe entre la evaluación de la gestión institucional y la calidad del servicio educativo en las isntituciones educativas del distrito de Huaral - Lima, 2018.

\section{MATERIALES Y MÉTODOS}

Al ser una investigación cuantitativa de nivel explicativa, se considera prudente aplicar un cuestionario para cada una de las variables de análisis.

Los resultados de esta investigación fueron en base a la aplicación de los instrumentos, que para los fines mencionados se consideró el cuestionario para ambas variables.

\section{RESULTADOS}

La investigación se apoyó del tratamiento estadístico, para ello se consideró el programa SPSS versión 21, cuyos resultados estadísticos permitieron la contrastación de las hipótesis, los cuales se muestran a continuación:

\section{Prueba de hipótesis general}

Ha. La Evaluación de la Gestión Institucional se relaciona significativamente con la Calidad del Servicio Educativo.

Ho. La Evaluación de la Gestión Institucional no se relaciona significativamente con la Calidad del Servicio Educativo.

Tabla 1

Correlación de la hipótesis general

\begin{tabular}{|c|c|c|c|}
\hline \multicolumn{2}{|c|}{ Correlaciones } & & \multirow{2}{*}{$\begin{array}{c}\begin{array}{c}\text { Calidad del } \\
\text { Servicio } \\
\text { Educativo }\end{array} \\
0,822\end{array}$} \\
\hline \multirow{3}{*}{$\begin{array}{l}\text { Rho de } \\
\text { pearman }\end{array}$} & \multirow{3}{*}{$\begin{array}{l}\text { Gestión } \\
\text { Institucional }\end{array}$} & $\begin{array}{l}\text { Coeficiente de } \\
\text { correlación }\end{array}$ & \\
\hline & & Sig. (bilateral) & 0,000 \\
\hline & & $\mathrm{N}$ & 101 \\
\hline
\end{tabular}

Interpretación: Con la aplicación de la fórmula estadística 
mostrada en el cuadro, con un margen de error al $5 \%$, se estableció una correlación muy buena de Rho $=0,822$ y el $p=0,000$, lo que nos permite dejar de lado la hipótesis nula y admitir la hipótesis alterna, cuyos resultados evidenciaron la insatisfacción mayoritaria de los estudiantes con respecto a la gestión institucional calificado de a veces adecuado, incidiendo directamente con la calidad de las instituciones en estudio considerado regular.

\section{Contrastación de la primera hipótesis específica}

Ha. La Motivación para el aprendizaje se relaciona significativamente con la Calidad del Servicio Educativo.

Ho. La Motivación para el aprendizaje no se relaciona significativamente con la Calidad del Servicio Educativo.

Tabla 2

Correlación de la primera hipótesis específica

\begin{tabular}{|c|c|c|c|}
\hline \multicolumn{3}{|c|}{ Correlaciones } & $\begin{array}{c}\text { Calidad del } \\
\text { Servicio } \\
\text { Educativo }\end{array}$ \\
\hline \multirow{3}{*}{$\begin{array}{l}\text { Rho de } \\
\text { Spearman }\end{array}$} & \multirow{3}{*}{$\begin{array}{c}\text { Motivación } \\
\text { para el } \\
\text { aprendizaje }\end{array}$} & $\begin{array}{l}\text { Coeficiente } \\
\text { de } \\
\text { correlación }\end{array}$ & $0,561^{* *}$ \\
\hline & & Sig. (bilateral) & 0,000 \\
\hline & & $\mathrm{N}$ & 101 \\
\hline
\end{tabular}

Interpretación: Con la aplicación de la fórmula estadística mostrada en el cuadro, con un margen de error al $5 \%$, se estableció una correlación moderada de Rho $=0,561$ y el $p$ $=0,000$, lo que nos permite dejar de lado la hipótesis nula y admitir la hipótesis alterna, cuyos resultados evidenciaron la insatisfacción mayoritaria de los estudiantes con respecto a la Motivación para el aprendizaje calificado de a veces adecuado, incidiendo directamente con la calidad de la instituciones en estudio considerados regular.

Contrastación de la segunda hipótesis específica

Ha. La Convivencia escolar se relaciona significativamente con la Calidad del Servicio Educativo.

Ho. La Convivencia escolar no se relaciona significativamente con la Calidad del Servicio Educativo.

Tabla 3

Correlación de la segunda hipótesis especifica

\begin{tabular}{|c|c|c|c|}
\hline \multicolumn{3}{|c|}{ Correlaciones } & $\begin{array}{c}\text { Calidad del } \\
\text { servicio } \\
\text { educativo }\end{array}$ \\
\hline \multirow{3}{*}{$\begin{array}{l}\text { Rho de } \\
\text { pearman }\end{array}$} & \multirow{3}{*}{$\begin{array}{c}\text { convivencia } \\
\text { escolar }\end{array}$} & $\begin{array}{l}\text { Coeficiente de } \\
\text { correlación }\end{array}$ & $0,640^{* *}$ \\
\hline & & Sig. (bilateral) & 0,000 \\
\hline & & $\mathrm{N}$ & 101 \\
\hline
\end{tabular}

Interpretación: Con la aplicación de la fórmula estadística mostrada en la tabla 3 , con un margen de error al $5 \%$, se estableció una correlación buena de Rho $=0,640$ y el $p=$ 0,000 , lo que nos permite dejar de lado la hipótesis nula y admitir la hipótesis alterna, cuyos resultados evidenciaron insatisfacción mayoritaria de los estudiantes con respecto a la convivencia escolar calificado de a veces adecuado, incidiendo directamente con la calidad del servicio ofrecido por las instituciones de la población de estudio referidos de regular.

\section{Contrastación de la tercera hipótesis específica}

Ha. La Cooperación con la identidad se relaciona significativamente con la Calidad del Servicio Educativo.

Ho. La Cooperación con la identidad no se relaciona significativamente con la Calidad del Servicio Educativo.

\section{Tabla 4}

Correlación de la tercera hipótesis especifica

\begin{tabular}{lccc}
\hline Correlaciones & \multicolumn{3}{c}{$\begin{array}{c}\text { Calidad del } \\
\text { servicio } \\
\text { educativo }\end{array}$} \\
\hline \multicolumn{3}{c}{$\begin{array}{c}\text { Coeficiente } \\
\text { de }\end{array}$} \\
$\begin{array}{l}\text { Rho de } \\
\text { Spearman }\end{array}$ & $\begin{array}{c}\text { Cooperación } \\
\text { con } \\
\text { identidad }\end{array}$ & $\begin{array}{c}\text { correlación } \\
\text { Sig. } \\
\text { (bilateral) } \\
\mathrm{N}\end{array}$ & $0,562^{* *}$ \\
\hline
\end{tabular}

Interpretación: Con la aplicación de la fórmula estadística mostrada en el cuadro, con un margen de error al $5 \%$, se estableció una correlación moderada de Rho $=0,562$ y el $p$ $=0,000$, lo que nos permite dejar de lado la hipótesis nula y admitir la hipótesis alterna, cuyos resultados evidenciaron insatisfacción mayoritaria de los estudiantes con respecto a la Cooperación con la identidad calificado de poco adecuado, incidiendo directamente con la calidad del servicio ofrecido en las instituciones del estudio considerado regular.

\section{Contrastación de la cuarta hipótesis específica}

Ha. La Política de inclusión está relacionado significativamente con la Calidad del Servicio Educativo.

Ho. La Política de inclusión no está relacionado significativamente con la Calidad del Servicio Educativo.

\section{Tabla 5}

Correlación de la cuarta hipótesis especifica

\begin{tabular}{lccc}
\hline \multicolumn{2}{l}{ Correlaciones } & \multicolumn{1}{c}{$\begin{array}{c}\text { Calidad del } \\
\text { servicio educativo }\end{array}$} \\
\hline Rho de & $\begin{array}{c}\text { Política } \\
\text { de }\end{array}$ & $\begin{array}{c}\text { Coeficiente } \\
\text { de correlación }\end{array}$ & $0,472^{* *}$ \\
Spearman & inclusión & Sig. (bilateral) & 0,000 \\
& & $\mathrm{~N}$ & 101 \\
\hline
\end{tabular}

Interpretación: Con la aplicación de la fórmula estadística mostrada en el cuadro, con un margen de error al $5 \%$, se estableció una correlación moderada de Rho $=0,472$ y el $p$ $=0,000$, lo que nos permite dejar de lado la hipótesis nula y admitir la hipótesis alterna, cuyos resultados evidenciaron la insatisfacción mayoritaria de los estudiantes con respecto a la Política de inclusión calificado de a veces adecuado, incidiendo directamente con la calidad del servicio de las escuelas analizadas considerados de regular.

\section{Contrastación de la quinta hipótesis específica}

Ha. El Clima institucional está relacionado de manera significativa con la Calidad del Servicio Educativo.

Ho. El Clima institucional no está relacionado de manera significativa con la Calidad del Servicio Educativo. 
Tabla 6

Correlación de la quinta hipótesis especifica

\begin{tabular}{|c|c|c|}
\hline Correlaciones & & $\begin{array}{l}\text { Calidad del } \\
\text { servicio } \\
\text { educativo }\end{array}$ \\
\hline \multirow{3}{*}{$\begin{array}{c}\text { Clima } \\
\text { institucional }\end{array}$} & $\begin{array}{l}\text { Coeficiente de } \\
\text { correlación }\end{array}$ & $0,757^{* *}$ \\
\hline & Sig. (bilateral) & 0,000 \\
\hline & $\mathrm{N}$ & 101 \\
\hline
\end{tabular}

Interpretación: Con la aplicación de la fórmula estadística mostrada en el cuadro, con un margen de error al $5 \%$, se estableció correlación buena de Rho $=, 757$ y el $p=0,000$, lo que nos permite dejar de lado la hipótesis nula y admitir la hipótesis alterna, cuyos resultados evidenciaron la insatisfacción mayoritaria de los estudiantes con respecto a la Clima institucional calificado de a veces adecuado, incidiendo directamente con la calidad del servicio de las instituciones educativas de estudio consideradas de regular.

Contrastación de la sexta hipótesis específica

Ha. La Participación se relaciona significativamente con la Calidad del Servicio Educativo.

Ho. La Participación no se relaciona significativamente con la Calidad del Servicio Educativo.

Tabla 7

Correlación de la sexta hipótesis especifica

\begin{tabular}{lcc}
\hline Correlaciones & Coeficiente & $\begin{array}{c}\text { Calidad del } \\
\text { servicio } \\
\text { educativo }\end{array}$ \\
\hline Rho de & Participación & $0,621^{* *}$ \\
Spearman & Sig. (bilateral) & 0,000 \\
& $\mathrm{~N}$ & 101 \\
\hline
\end{tabular}

Interpretación: Con la aplicación de la fórmula estadística mostrada en el cuadro, con un margen de error al 5\%, se estableció una correlación buena de Rho $=0,621$ y el $p=$ 0,000 , lo que nos permite dejar de lado la hipótesis nula y admitir la hipótesis alterna, cuyos resultados evidenciaron la insatisfacción mayoritaria de los estudiantes con respecto a la Participación calificado de a veces adecuado, incidiendo directamente con la calidad del servicio de las instituciones en análisis considerados regular.

\section{DISCUSIÓN}

Este estudio científico analizó la relación entre la Evaluación de la Gestión Institucional y la Calidad del Servicio Educativo en el contexto educativo de las Instituciones Educativas del distrito de Huaral - Lima, 2018

Para empezar, se encontró en la hipótesis general una correlación muy buena de Rho $=0,822$ representado por la Gestión Institucional, identificado mayoritariamente por los estudiantes como a veces adecuado por un $62,4 \%$, lejos de un $21,8 \%$ que lo describen como adecuado, y la Calidad del Servicio Educativo que a perspectiva del $60,4 \%$ de los docentes tienen una calificación regular, seguido del $27,7 \%$ como buena. Evidenciando que las acciones institucionales que toman las autoridades no cumplen satisfactoriamente con las expectativas de los estudiantes lo cual disminuye las posibilidades de progreso en la calidad del servicio que ofrecen las instituciones analizadas

La hipótesis específica 1 estableció una correlación moderada de $\mathrm{Rho}=0,561$ entre la dimensión Motivación para el aprendizaje, identificado mayoritariamente por los estudiantes como a veces adecuado por un $55,4 \%$, lejos de un $34,7 \%$ que lo describen como adecuado, y la Calidad del Servicio Educativo que a criterio del $60.4 \%$ de los docentes tienen una calificación regular, seguido del 27,7\% como buena. Encontrándose que las acciones de perseverancia en el cumplimiento de tareas educativas no cumplen satisfactoriamente con las expectativas de los estudiantes lo cual disminuye las posibilidades de progreso en la calidad del servicio educativo.

La hipótesis específica 2 estableció una correlación buena de $\mathrm{Rho}=0,640$ entre la dimensión Convivencia escolar, identificado mayoritariamente por los estudiantes como a veces adecuado por un $52,5 \%$, lejos de un $27,7 \%$ que lo describen como adecuado, y la Calidad del Servicio Educativo que a consideración del $60,4 \%$ de los docentes tienen una calificación regular, seguido del $27,7 \%$ como buena. Resolviéndose que el cuidado del patrimonio, cortesía y compañerismo no cumplen satisfactoriamente con las expectativas de los estudiantes lo cual disminuye las posibilidades de progreso en la calidad del servicio educativo.

La hipótesis específica 3 identificó una correlación moderada de Rho $=0,562$ entre la dimensión Cooperación con entidades productivas, descrito mayoritariamente por los estudiantes como poco adecuado por un $36,6 \%$, seguido de un $36,6 \%$ como a veces adecuado, y la Calidad del Servicio Educativo que a consideración del $60,4 \%$ de los docentes tienen una calificación regular, seguido del $27,7 \%$ como buena. Resolviéndose que la gestión con respecto a la gestión que las autoridades ejercen sobre los comercios, empresas, municipalidad, entre otras instituciones que se vinculan a la institución de estudio no cumplen satisfactoriamente con las expectativas de los estudiantes lo cual disminuye las posibilidades de progreso en la calidad del servicio educativo.

La hipótesis específica 4 encontró una correlación moderada de Rho $=0,472$, entre la dimensión Política de inclusión, señalado mayoritariamente por los estudiantes como a veces adecuado por un $49,5 \%$, seguido de un $26,7 \%$ como poco adecuado, y la Calidad del Servicio Educativo que a perspectiva del $60,4 \%$ de los docentes tienen una calificación regular, seguido del 27,7\% como buena. Encontrándose que el apoyo de la institución en cuanto a las limitaciones físicas, los problemas de aprendizaje, problemas disfuncionales en la familia no cumplen satisfactoriamente con las expectativas de los estudiantes lo cual disminuye las posibilidades de progreso en la calidad del servicio de las instituciones seleccionados para esta investigación.

La hipótesis específica 5 halló una correlación buena de Rho $=0,757$ entre la dimensión Clima institucional encontrado mayoritariamente por los estudiantes como a veces adecuado por un $54,4 \%$, lejos de un $26,7 \%$ como adecuado, y la Calidad del Servicio Educativo que a perspectiva del $60,4 \%$ de los docentes tienen una calificación regular, seguido del $27,7 \%$ como buena. Identificando que la gestión de las normas, reglas, políticas y procedimientos aplicados en la institución no cumplen satisfactoriamente con las expectativas de los estudiantes lo cual disminuye las posibilidades de mejora en la calidad del servicio educativo 
La hipótesis específica 6 encontró una correlación buena de Rho $=0,621$ entre la dimensión Participación encontrado mayoritariamente por los estudiantes como a veces adecuado por un $40.6 \%$, seguido de un $40.6 \%$ como adecuado, y la Calidad del Servicio Educativo que a opinión del $60.4 \%$ de los docentes tienen una calificación regular, seguido del $27.7 \%$ como buena. En otras palabras, notamos que la participación en eventos culturales, deportivos o similares de no son fomentados con idoneidad no cumplen satisfactoriamente con las expectativas de los estudiantes lo cual disminuye las posibilidades de progreso en la calidad del servicio educativo.

Luego del análisis estadístico, se contrastó con los de Asprella (2015) quien analiza la gestión institucional desde una perspectiva directiva, donde destaca la importancia del análisis situacional cuyos planteamientos deben ser consecuencias de las ideas y percepciones que transitan el ámbito educativo. En este punto se debe detallar que los directivos son quienes guían las gestiones que se realizan en la institución, sin embargo, también es responsabilidad de recoger opiniones entre el personal administrativo y docente, comunicación debe ser constante y analizar dichas opiniones, puesto que es necesario que organizar todas las medidas necesarias para que el estudiante se sienta motivado, experimente una buena convivencia, se sienta incluido, etc. En otras apalabras, las autoridades no solo deben analizar la forma de gestión, sino actuar y corregir dichas formas.

En los resultados de Elliot (2017) se identificaron una correlación entre la gestión educativa estratégica y la calidad de servicio educativo, este último expresado en el desempeño administrativo, capacitación docente, coordinación y participación administrativa, infraestructura y equipamiento. En otras palabras, se dan cuenta que, si se identifica las verdaderas necesidades de los estudiantes, identificando puntos de mejora la calidad del servicio se hace evidente. Sin embargo, también debemos tener en cuenta que no solo son materiales y docentes, ya que así estudiantes con la mejor infraestructura, o equipos de última generación, si es que el estudiante no está motivado y se fortalezca un clima idóneo de buen compañerismo, dimensiones que tratamos en nuestro estudio, la calidad no podrá ser bien apreciada.

\section{CONCLUSIÓN}

La gestión institucional en la isntituciones educativas evaluadas no cumplen satisfactotiamante con las espectativas de los estudiantes, lo cual disminuye las posibilidades de progreso en la calidad de servicio.

\section{REFERENCIAS BIBLIOGRÁFICAS}

Areche, V. (2013). La gestión institucional y la calidad en el servicio educativo según la percepción de los docentes y padres de familia del $3^{\circ}, 4^{\circ}$ y $5^{\circ}$ de secundaria del colegio "María Auxiliadora" de Huamanga-Ayacucho, 2011 (Tesis Posgrado) Universidad Nacional Mayor de San Marcos, Lima, Perú.

Asprella, G. (2015). Modelos de gestión en Directivos de instituciones educativas de nivel secundario (Tesis Posgrado) Universidad de Sevilla, España. Recuperado en: https://idus.us.es/xmlui/bitstream/ handle/11441/39743/Tesis\%20Gabriel\%20 Asprella.\%20Versi\%C3\%B3n\%20impresa. pdf?sequence $=1$

Consejo Nacional de Educación (2016). Encuesta Nacional a Docentes de Instituciones Educativas Estatales y No Estatales, Lima, Perú. Recuperado en: http:// repositorio.minedu.gob.pe/handle/123456789/4872

Elliot, J. (2017). La gestión educativa estratégica y la calidad del servicio educativo en las Instituciones Educativas del nivel secundario de la Provincia Paucar del Sara - Ayacucho (Tesis Posgrado) Universidad Nacional de Educación, Lima, Perú.

Escale (2017). Entorno de enseñanza. MINEDU, Perú. Recuperado en: http://escale.minedu.gob.pe/ ueetendencias2016

Guadalupe, C. et al. (2017). Estado de la Educación en el Perú. Análisis y perspectivas de la educación básica. Lima: Editorial GRADE.

IPEBA (2014). ¿Qué y cómo evaluamos la gestión de la institución educativa? Lima: Sineace.

Perú entre los países que menos invierten en educación, por debajo de los US\$50,000. (12 de marzo de 2018). Gestión. Recuperado en: https://gestion.pe/ economia/peru-paises-invierten-educacion-debajous-50-000-229121

Ropa, B. (2014) Administración de la calidad en los servicios educativos. Horizonte de la Ciencia. 4 (6) p. 67-73 